\title{
THE RESILIENCE OF ONCOLOGISTS DURING THE EARLY TIME OF THE COVID-19 PANDEMIC
}

\author{
A. Lasagna ${ }^{1}$, S. Secondino ${ }^{1}$, F. Agustoni ${ }^{1}$, T. Monaco ${ }^{1}$, I. Imarisio ${ }^{1}$, A. Pagani ${ }^{1}$, \\ G. Rizzo ${ }^{1}$, R. J. Tancredi ${ }^{1}$, E. Pozzi ${ }^{1}$, E. Ferraris ${ }^{1}$, S. Chiellino ${ }^{1}$, C. Gandini ${ }^{1}$, \\ A. Ferrari ${ }^{1}$, S. G. Brugnatelli ${ }^{1}$, P. Pedrazzoli ${ }^{1,2}$ \\ ${ }^{1}$ Medical Oncology Unit, Fondazione IRCCS Policlinico San Matteo, Pavia, Italy \\ ${ }^{2}$ Department of Internal Medicine and Medical Therapy, University of Pavia, Pavia, Italy
}

\section{CORRESPONDING AUTHOR:}

\author{
Angioletta Lasagna \\ Medical Oncology Unit \\ Fondazione IRCCS Policlinico San Matteo \\ piazzale C. Golgi 19 \\ 27100 Pavia, Italy \\ E-mail: a.lasagna@smatteo.pv.it \\ ORCID: 0000-0002-9611-1164
}

Doi: $10.48286 / a r 0.2021 .07$

History

Received: Dec 5, 2020

Accepted: Feb 3, 2021

Published: Mar 1, 2021

\begin{abstract}
With the emergence of COVID-19, Oncologists have had to face up to the challenge of continuing active treatments without compromising patients and healthcare personnel's safety. We introduced a double-step triage strategy (by phone and on site) for cancer patients in order to identify patients at risk of COVID-19 and to avoid their admission to our Oncology Unit.

From February $24^{\text {th }}$ to April $7^{\text {th }} 2020$, we performed 819 phone calls, leading to the authorization of 788 accesses (312 patients) to the outpatient clinic. 26 patients were kept at home, 23 managed with
\end{abstract}

\section{KEY WORDS}

COVID-19; cancer; triage; resilience; management strategy. symptomatic treatments and 3 hospitalized for suspected COVID-19. At the second triage level, 5 patients weren't admitted to the Outpatient clinic for respiratory distress. None of the 58 healthcare workers were infected by SARS-CoV-2.

The surveillance strategy was carried on according to hospital indications which opted to do the screening only of people reporting symptoms because an active strategy was not feasible at that time. Our practical approach allows the identification of patients at risk of COVID-19 infection and appears effective in maintaining cancer care with high levels of safety.

\section{IMPACT STATEMENT}

A simple double-step triage strategy has been useful in maintaining cancer patient and health care worker safety during COVID-19 emergency in Italy. 


\section{INTRODUCTION}

Italy's COVID-19 outbreak originated in Southwest Lombardy, on February $21^{\star}, 2020$. The Fondazione IRCCS Policlinico San Matteo in Pavia, the nearest and largest teaching hospital near Codogno, was involved in the management of the outbreak from the start, undergoing a rapid and thorough reorganization $(1,2)$. In this emergency, the behavior of health care workers had to follow the risk management strategy of "resilience", a term used to define the ability to face a new situation by improving management skills (3). Also the oncologist community suggested to adapt the patient management in order to assist cancer patients in the safest way by introducing methods to improve a careful evaluation of every single patient to optimize oncological treatment (4). Practically, the oncologists needed to perform a substantial quality improvement focused to avoid nosocomial COVID 19 spread. According to these suggestions, and moving from a Deming cycle (Plan - Do - Check Act), we planned $(P)$ and put into practice a simple and safe triage protocol (D) aimed to screen each patient before his admission into Day Hospital or Ward spaces which had to remain COVID-19 free. This paper describes the obtained results (Check) to verify which improvements are needed $(A)$.

\section{MATERIALS AND METHODS}

The outbreak of COVID-19 required an immediate reorganization of our workflow to minimize the risk of contamination. Before this emergency, there were no specific procedures to evaluate patients before admission for active treatment and there were no epidemiological checks.

From February $24^{\text {th }}$, we chose to start a protocol based on a double-step triage strategy for cancer patients, already under treatment or newly diagnosed, consisting of:

- first step: a phone call the day before active therapy or admission;

- second step: a clinical evaluation before the admission to the outpatient and inpatient wards on the day of the treatment.

The phone call was done by an experienced clinician the morning before the scheduled access in order to evaluate the clinical conditions of the patient and of the members of his/her family by asking information about the presence of signs/ symptoms as detailed in table I.

Every call lasted about 15 minutes and was preferably directed to the patient and not to the caregiver or to a member of the family to make sure that the symptoms were really reported and not missed.

This assessment took account of signs and symptoms potentially related to the underlying disease or treatment toxicity.

Moreover, the clinician asked each patient if, within the previous 72 hours, he/she had been into known outbreak areas (for the first period of epidemic), or had had direct contact with people known to have been affected by COVID-19 or with people currently in quarantine. The same questions were addressed also to the patient's relatives, to identify potentially infectious close contacts.

The questionnaire was modified according to relevant information on COVID-19 published in the medical literature (e.g., after the alert on anosmia and dysgeusia as consequence of COVID-19 (5)) and to the local protocol management (6).

In the presence of symptoms potentially related to COVID-19 infection, the patient was invited to stay at home, and a symptomatic treatment was suggested. Daily phone monitoring was implemented and, in cases of worsening of clinical status, the patient was signaled to the general practitioner for clinical evaluation at home and eventually referred to the regional Emergency Medical System (EMS) for evaluation for hospital admission (7), according to standard protocols of outpatient management. The second triage level was performed before the patient entered either the day hospital or the inpatient ward by the nurse case manager and a physician, both wearing personal protection equipment (PPE) as suggested by WHO guidelines (8). This triage consisted of a new evaluation of clinical state by measuring body temperature and evaluating possible signs and symptoms of respiratory infections. This procedure aimed at a more careful examination of patients to reinforce what emerged at the first triage level. Both in the triage area and in the therapy rooms, a security distance of at least $120 \mathrm{~cm}$ was rigorously observed, and every patient was trained to wear a surgical mask and shoe covers, and to disinfect, at least at admission and before discharge, the hands with an hydro-alcoholic gel. Patients who were hospital admitted, in case of fever or other suspicious symptoms, underwent 
Temperature $\geq 37.3^{\circ} \mathrm{C}$

Gastrointestinal Symptoms (nausea/vomiting/diarrhea)

Respiratory symptoms

(cough/nasal discharge, dyspnea)

Anosmia

Ageusia

Asthenia

Conjunctivitis

Headache

Myalgia/arthralgia

Patients' relatives with same symptoms

\section{NUMBER OF PATIENTS REPORTING SIGNS/SYMPTOMS}

Patients with 1 sign/symptom n. 15

Patients with 2-3 signs/symptoms n. 10

Patients with > 3 signs/symptoms n. 1 (Fever, Cough, Anosmia)

Symptomatic patients' relatives n. 1 (Fever)

Table I. First step triage questions asked by phone to patients the day before admission and number of patients reporting signs/symptoms.

a nasopharyngeal swab for SARS-CoV 2, an X-ray of the thorax and blood exams. In case the swab was negative, but X-ray was doubtful for a diagnosis of interstitial pneumonia, patients were not allowed to entry in the ward. With the aim of reducing social interaction, patient's relatives were not allowed to enter the hospital area.

Healthcare workers at the second level triage position and involved in the direct care of patients used the WHO-suggested PPE: eye protection (goggles), liquid-repelling gowns, double gloves, a class-2 filtering face-piece respirator (FFP2). Workers inside the Day Hospital room were equipped with surgical mask, goggles, not waterproof gowns and, obviously, gloves. The aim was to supply each worker with standard protective equipment for each work-shift. Cleaning procedures have been also implemented and standardized; in particular, ward surfaces were cleaned every day with sodium hypochlorite in terminal sanitation $(9,10)$. Every day, a careful check of the procedure was made, with the aim of revealing any deviation from the protocol. Furthermore, the people wearing PPE followed refresher-training sessions on their use. The study was approved by the local Ethics Committee (Comitato Etico Area Pavia) and Institutional Review Board (P-20200038244), in accordance with the ethical standards established in the Declaration of Helsinki of 1946. All the subjects signed an informed written consent before the enrollment in the study. This brief report has been written following SQUIRE 2.0 framework as suggested by quality improvement guidelines.

\section{RESULTS}

From February $24^{\text {th }}$ to April $7^{\text {th }} 2020$, we have performed 819 phone calls, leading to the authorization of 788 accesses (312 patients) to the outpatient clinic for active treatments. In the same period, one year ago, without any specific triage procedure, we recorded 820 Day hospital accesses. Twenty-six patients $(8.3 \%)$ with fever $\left(>37.3^{\circ} \mathrm{C}\right)$ and/or other symptoms were kept at home and managed by repeated telephone calls; 3 patients were subsequently hospitalized for suspected COVID-19, while 23 were managed at home with symptomatic treatments and antibiotics.

At the second triage level, 5 patients presented persistent fever or respiratory distress before be- 
ing admitted to the Outpatient clinic, 2 of them were tested PCR-positive.

Overall, 5 triaged patients ( 3 at the first step and 2 at the second one) were hospitalized and proved positive for SARS-CoV-2 by nasal swab. All patients experienced a course of hospital stay, treated with steroids, heparin and antibiotics, and were discharged alive.

In the same period 177 patients were admitted to the inpatient ward: 89 for cancer treatments, including 5 with germ cell tumors undergoing stem cell transplantation programs, 88 for diagnostic procedures or for supportive measures. As of April $7^{\text {th }}$, none was found to be COVID-19-positive and both outpatient and inpatient areas were still COVID-19 free. Moreover, no healthcare workers became infected by SARS-CoV-2.

The healthcare staff consisted of 20 doctors and 38 nurses and other people dedicated to patient assistance. The surveillance strategy was carried on according to hospital indications which opted to do the screening of people reporting symptoms because an active strategy was not feasible at that time.

The main characteristics of the patients stopped at the two triage levels are shown in the table II.

\section{DISCUSSION}

During the COVID-19 pandemic, oncologists were between Scylla and Charybdis (11): they had to protect both patients and themselves against the risk of infection and, at the same time, allow their patients to receive appropriate diagnostic workups and curative therapies (12).

So far many authors have reported the importance of organizing patient flow, including adopting the strategy of telemedicine, to minimize the contact between them (13-15). The approach described in the present single-institution experience, based on a simple double-step triage strategy, allows the identification of patients at risk for active COVID-19 infection, and avoids their admission to the outpatient clinic and inpatient ward. As compared with the pre-screening protocol, we arranged our activity without substantially reducing the normal "pre-COVID" level, and without the need of extra resources. During this emergency some health activities were suspended or drastically reduced, such as the follow-up visits which represent a significant commitment "in peacetime" in terms of time worked. Also first contact visits diminished as consequence of the reduction of surgical and diagnostic procedures.

\begin{tabular}{|c|c|c|}
\hline TYPE OF TUMOR & NUM PATIENTS & $\%$ \\
\hline Lung Cancer & 11 & 36 \\
\hline Breast cancer & 6 & 20 \\
\hline Colon cancer & 6 & 20 \\
\hline Melanoma & 2 & 6 \\
\hline Gastric cancer & 1 & 3 \\
\hline Pancreatic cancer & 1 & 3 \\
\hline Prostate cancer & 1 & 3 \\
\hline Thymic carcinoma & 1 & 3 \\
\hline Ovaric cancer & 1 & 3 \\
\hline Renal cancer & 1 & 3 \\
\hline $\begin{array}{c}\text { TYPE OF ONCOLOGICAL } \\
\text { TREATMENT }\end{array}$ & NUM PATIENTS & $\%$ \\
\hline Chemotherapy & 24 & 78 \\
\hline Immunotherapy & 5 & 16 \\
\hline Targeted therapy & 2 & 6 \\
\hline SETTING OF TREATMENT & NUM PATIENTS & $\%$ \\
\hline Neoadjuvant & 0 & 0 \\
\hline Adjuvant & 5 & 16 \\
\hline Metastatic & 26 & 84 \\
\hline COMORBIDITIES & NUM PATIENTS & $\%$ \\
\hline Hypertension & 16 & 52 \\
\hline Type 2 Diabetes & 6 & 19 \\
\hline BPCO/Asthma & 4 & 13 \\
\hline Coronaric heart disease & 4 & 13 \\
\hline Obesity & 3 & 10 \\
\hline $\mathrm{HCV} / \mathrm{HBC}$ & 2 & 6 \\
\hline HIV & 0 & 0 \\
\hline No comorbitidies & 10 & 32 \\
\hline$\geq 1$ comorbidity & 21 & 68 \\
\hline SEX & NUM PATIENTS & $\%$ \\
\hline Male & 18 & 58 \\
\hline Female & 13 & 42 \\
\hline AGE (YEARS) & NUM PATIENTS & $\%$ \\
\hline $18-29$ & 0 & 0 \\
\hline $30-39$ & 1 & 3 \\
\hline $40-49$ & 5 & 16 \\
\hline $50-59$ & 9 & 29 \\
\hline $60-69$ & 8 & 26 \\
\hline $70-79$ & 7 & 23 \\
\hline$\geq 80$ & 1 & 3 \\
\hline
\end{tabular}

Table II. Patients' characteristics not admitted in Day Hospital (first step and second step).

Some very frail patients were hospitalized to receive active oncological treatments or invasive diagnostic procedures and to avoid to expose them to COVID-19 infection during their stay in various areas of the hospital.

This study has main limitations, consisting in the 
lack of a control and of the possibility to compare it with the approach of other Hospitals in the same area: some Hospitals became COVID at all and the Hospitals that remained COVID free adopted completely different protocols. Moreover, we did not put in place protocols like telemedicine; in fact they were reserved to the management of patients remaining at home and were not enough to screen patients needing a hospital access.

\section{CONCLUSIONS}

Our approach put successfully into practice the capacity to adapt our management to a global health emergency ("resilience") and to maintain high levels of safety for patients and health care workers as demonstrated by the absence of COVID-19 infections among patients and health care workers. According to the guidelines of improving quality in healthcare, this kind of strategy is fully sustainable even in a setting where the availability of swab and serologic tests is limited. Moreover, even where diagnostic methods are available, combining a clinical and virologic approach may be useful in case of other pandemic waves when the capacity of the healthcare services may be once again overcrowded.

\section{ACKNOWLEDGEMENTS}

We are indebted with Professor Raffaele Bruno and the medical staff of Infectious Diseases Unit for the cooperation in organizing the management strategy.

\section{ETHICS}

\section{Fundings}

This work was partially supported by Ricerca Corrente grant no 08067619, Fondazione IRCCS Policlinico San Matteo.

\section{CONFLICTS OF INTERESTS}

The authors have declared no conflict of interests.

\section{Availability of data and material}

The data underlying this article can be shared just before a reasonable request to the corresponding author.

\section{Code availability \\ N/A}

\section{Authors' contribution}

AL: conception of the work, drafting and writing the work; SS, FA, TM, II, AP, GR, RJT, EP, EF, SC, CG, AF: acquisition, analysis and interpretation of data for the work; SGB and PP: revising the work critically for important intellectual content.

All authors contributed to the article and approved the submitted version.

\section{Ethical approval and consent to participate}

The study was approved by the local Ethics Committee (Comitato Etico Area Pavia) and Institutional Review Board (P-20200038244), in accordance with the ethical standards established in the Declaration of Helsinki of 1946. All the subjects signed an informed written consent before the enrollment in the study. 


\section{REFERENCES}

1. Asperges E, Novati S, Muzzi A, et al. Rapid response to COVID-19 outbreak in Northern Italy: how to convert a classic infectious disease ward into a COVID-19 response centre. J Hosp Infect 2020;105(3):477-9

2. Perlini S, Canevari F, Cortesi S, et al. Emergency Department and Out-of-Hospital Emergency System (112-AREU 118) integrated response to Coronavirus Disease 2019 in a Northern Italy centre. Intern Emerg Med 2020;15(5):825-33.

3. Mitchell T, Harris K. Resilience: A risk management approach. Available at: https://www.odi.org/sites/ odi.org.uk/files/odi-assets/publications-opinion-files/7552.pdf Accessed: 07/04/2020.

4. Italian Association of Medical Oncology Statement. Available at: https://www.aiom.it/comunicato-aiom-per-emergenza-corona-virus/. Accessed: 07/04/2020.

5. Lechien JR, Chiesta-Estomba CM, De Siati DR, et al. Olfactory and gustatory dysfunctions as a clinical presentation of mild-to-moderate forms of the coronavirus disease (COVID-19): a multicenter European study. Eur Arch Otorhinolaryngol 2020;277(8):2251-61.

6. Fondazione IRCCS Policlinico San Matteo. COVID Management Guidelines. Available at: http:// intranet.sanmatteo.org/site/home/sistema-qualita/protocolli-aziendali/mcs---modelli-comportamentali-standardizzati/docCatdocumenti-gestione-covid-19.1001181.1.50.1.all. html Accessed: 07/04/2020.

7. Spina S, Marrazzo F, Migliari M, Stucchi R, Sforza A, Fumagalli R. The response of Milan's emergency medical system to the COVID-19 outbreak in Italy. Lancet 2020;395(10227):e49-e50.
8. WHO. Rational use of personal protective equipment (PPE) for coronavirus disease (COVID-19). Available at: https://apps.who.int/ iris/bitstream/handle/10665/331498/WHO2019-nCoV-IPCPPE_use-2020.2-eng.pdf Date: March 19, 2020. Accessed: 10/04/2020.

9. Colaneri $M$, Seminari E, Piralla A, et al. Lack of SARS-CoV-2 RNA environmental contamination in a tertiary referral hospital for infectious diseases in Northern Italy. J Hosp Infect 2020;105(3):474-6.

10. European Centre for Disease Prevention and Control (ECDC) Technical Report. Disinfection of environment in healthcare and non-healthcare settings potentially contaminated with SARS-COV-2. March 2020.

11. Lewis MA. Between Scylla and Charybdis - Oncologic Decision Making in the Time of Covid-19. N Engl J Med 2020;382(24):2285-7.

12. Tondo L. Life 'hanging by a thread' for Italian cancer patients in coronavirus crisis. The Guardian 7 Apr 2020.

13. Hanna TP, Evans GA, Booth CM. Cancer, COVID-19 and the precautionary principle: prioritizing treatment during a global pandemic. Nat Rev Clin Oncol 2020;17(5):268-70.

14. Lambertini M, Toss A, Passaro A, et al. Cancer care during the spread of coronavirus disease 2019 (COVID-19) in Italy: young oncologists' perspective. ESMO Open 2020;5(2):e000759.

15. Liu R, Sundaresan $T$, Reed ME, Trosman JR, Weldon CB, Kolevska T. (2020) Telehealth in Oncology During the COVID-19 Outbreak: Bringing the House Call Back Virtually. JCO Oncol Pract 2020;16(6):289-293. 